\title{
¿Qué es y qué no es un dispositivo? Profanación y veridicción
}

\author{
Edgardo Castro ${ }^{1}$ \\ Conicet (Argentina)
}

Resumen: El presente artículo analiza la conferencia de Giorgio Agamben de 2005 titulada "Che cos'è un dispositivo?". Discute la filiación hegeliana del término "positividad” en Foucault y la genealogía teológica de los dispositivos sostenida por Agamben. Subrayando, respecto de estas dos cuestiones, las distancias entre el pensamiento de Agamben y el de Foucault. En la parte final, en cambio, se ocupa de las posibles convergencias entre la noción agambeniana de profanación y la foucaultiana de dispositivo.

Palabras clave: Dispositivo, Agamben, Foucault, Positividad, Profanación, Veridicción.

“Che cos’è un dispositivo?” se titula una conferencia de Giorgio Agamben del 2005, publicada en Italia el año siguiente como un pequeño libro. Aunque el autor no haga ninguna referencia al respecto, resulta casi evidente vincularla con la conferencia homónima de 1988, el último homenaje que hiciera Gilles Deleuze a su amigo Michel Foucault, fallecido pocos años antes. ${ }^{2}$ Podría decirse, incluso, que se trata de la repetición de un mismo gesto: retomar uno de los términos más representativos de Foucault para elaborar una interpretación que permita caracterizar todo su pensamiento. En efecto, para Deleuze, toda la filosofía de Foucault es vista finalmente como una filosofía de los dispositivos (Deleuze, 2003, p. 320). Pero Agamben va mucho más lejos. Por un lado, sus consideraciones exceden los límites del archivo foucaultiano. De acuerdo con sus propias palabras, su propósito es llevar a cabo "una

\footnotetext{
${ }^{1}$ Edgardo Castro nació en 1962. Es doctor en Filosofía por la Universidad de Friburgo (Suiza), actualmente es Profesor Titular ordinario de la asignatura Historia de la filosofía contemporánea en la Universidad Nacional de San Martín (Argentina) e Investigador del Consejo Nacional de Investigaciones Científicas y Técnicas. Ha sido docente en distintas universidades argentinas, y profesor invitado en el Istituto Italiano di Scienze Umane de Nápoles, en la Universidad Federal de Santa Catarina (Brasil), en la Universidad de Chile y en la Universidad Nacional de Colombia.

${ }^{2}$ Esta intervención fue publicada primero, pero parcialmente, en el Magazine Littéraire, en 1988 como "Qu'estce que un dispositif?". La versión completa, acompañada de un resumen de la discusión subsiguiente, apareció luego en la obra que reúne las exposiciones del encuentro en cuyo marco tuvo lugar (Michel Foucault Philosophe. Rencontre internationale, Paris, 9, 10, 11 janvier 1988, Paris, Seuil, 1989, pp. 185-195). Posteriormente, esta versión completa pero sin el resumen de la discusión, fue reimpresa en la compilación de textos y conversaciones de Deleuze, preparada por David Lapoujade (Deux régimes des fous, París, de Minuit, 2003 , pp. 316-325). Y una versión en inglés de este texto ha sido incluida, precisamente para explicar qué es un dispositivo, en la voz "Apparatus" del Cambridge Foucault Lexicon de L. Lawlor y John Nale (Cambridge University Press, New York, 2014).
} 
genealogía de este término [dispositivo] dentro de la obra de Foucault y luego en un contexto histórico más amplio", ${ }^{3}$ que resulta ser, como veremos, la historia de la teología (Agamben, 2006, p. 8). Por otro lado, retomando nuevamente sus palabras, puesto que en un determinado momento el interprete debe "abandonar el texto que está analizando y proceder por cuenta propia" (Agamben, 2006, 21), Agamben propone también una ontología y una política de los dispositivos.

Es posible resumir en cinco grandes tesis el recorrido de esta conferencia de Agamben: 1) En el pensamiento de Foucault, el término "dispositivo" vino a ocupar el lugar que tenía en sus investigaciones previas la noción de positividad, que le habría llegado desde Hegel a través de Jean Hyppolite (Agamben, 2006, p. 8-9). 2) Con el término dispositivo, entonces, Foucault se refiere a lo que Hegel llamaba positividad (p. 12). 3) Dado que el término latino "dispositio" ha sido la traducción del griego "oikonomía", en los dispositivos foucaultianos nos encontramos con la herencia teológica del término (p. 18). 4) Todo lo que existe puede ser dividido entre dos grandes categorías: substancias y dispositivos (p. 21). Y 5) la profanación es el contradispositivo (p. 28).

En el presente trabajo, nos proponemos desandar el camino de Agamben, en lugar de ir de Foucault a Agamben, haremos el recorrido inverso, de Agamben a Foucault. De esto modo, trataremos de establecer el alcance de las primeras tres tesis, de carácter filológicohermenéutico, para abordar luego la última, de corte más bien filosófico-político. Respecto de las primeras, nuestra intención es mostrar la distancia que, a nuestro modo de ver, finalmente subsiste entre ambos pensadores. A propósito de la última, en cambio, nos interesa mostrar las convergencias entre la noción agambeniana de profanación y la foucaultiana de veridicción.

\section{Positividad}

Es cierto, como sostiene Agamben en su conferencia, que el término "dispositivo" aparece en la obra de Foucault a mediados de la década de 1970 (2006, p. 5). En sus libros publicados en vida, exactamente en 1975 en Surveiller et punir. Reaparecerá luego en la obra del año inmediatamente sucesivo, La Volonté savoir. En sus libros, subrayamos, no es utilizado ni antes ni después, su vigencia se limita a estos dos años. Si extendemos sin

\footnotetext{
${ }^{3}$ Remitimos a las ediciones en lengua original. Todas las traducciones son nuestras. 
embargo nuestra mirada, hasta incluir todo el material publicado póstumamente, en particular sus cursos, el término hace su aparición en la lección del 7 de noviembre de 1973 de Le Pouvoir psychiatrique. A partir de ese momento y hasta el curso de 1979, Naissance de la biopolitique, es utilizado con frecuencia, precisamente, como señala también Agamben (2006, pp. 5-6), en el marco del estudio de lo que Foucault denominó la gubernamentalidad. Pero luego de la Naissance de la biopolitique su importancia disminuye. ${ }^{4}$ Respecto del uso foucaultiano del término "positividad" (positivité) el panorama es diferente; con mayor o menor frecuencia y con diferentes sentidos ha estado presente en los escritos de Foucault, teniendo en cuenta el estado actual de las publicaciones, desde el año 1957 en que hace su aparición.

Ahora bien, en cuanto concierne al sentido o los sentidos que ha tenido el término "positividad" en la obra de Foucault, es necesario distinguir al menos dos grupos: aquel con el que ha sido utilizado antes de Les Mots et les choses (1966) y L'Archéologie de savoir (1969) y el que ha adquirido a partir de estas obras, sobre todo en la última donde se convierte en un término propiamente técnico de su vocabulario. Así, si recorremos los escritos entre 1957 y 1966, nos encontramos con una serie de expresiones que podemos ejemplificar con las referencias retomadas a continuación. Refiriéndose a la cibernética en relación con la psicología (se trata de la primera aparición del término), Foucault afirma que "su positividad [la de la cibernética] parece alejarse de toda especulación" (1994, vol. 1, p. 136). En el mismo trabajo, pero esta vez a propósito de la investigación en el campo de la psicología, sostiene que "más allá de todo postulado especulativo ella está cargada de una positividad inmediata y, si la investigación se inscribe frecuentemente en un contexto positivista, si ella remite constantemente a una práctica real por oposición a la psicología filosófica, es precisamente porque quiere ser la demostración de una práctica posible” (1994, vol 1, p. 147). En la Histoire de la folie, hablando de la experiencia de la locura, afirma "el Renacimiento ha despojado a la miseria de su positividad mística" (1961, p. 80). En esta misma obra, a propósito de la relación entre el hombre y la animalidad, se habla de una "positividad natural" (1961, p. 202). Y, más adelante, esta vez refiriéndose a los posibles modos de concebir la historia de la locura, se sirve de la expresión "estilo de positividad" para hablar de aquello que es supuesto por todo positivismo, esto es, que para el reconocimiento de la locura como

\footnotetext{
${ }^{4}$ Acerca de la frecuencia del término "dispositivo" en los escritos de Foucault, cf. Castro, 2011, p. 114.
} 
realidad patológica, era necesario separarla del mundo moral en el que la había encerrado el clasicismo (1961, p. 208). A partir de Les Mots et les choses y, como ya señalamos, en particular de L'Archéologie du savoir, el término "dispositivo" adquiere en Foucault un sentido propiamente técnico dentro de su vocabulario, convirtiéndose en sinónimo de "a priori histórico", es utilizado para referirse al régimen discursivo al que pertenece un determinado saber y, por lo tanto, determina sus condiciones enunciativas (1969, p. 167). En resumen, podemos distinguir, entonces, tres sentidos del término: 1) como lo opuesto a la mera especulación, 2) como lo opuesto a la negatividad y 3) como equivalente a a priori histórico. El primero y el último sentido revisten una connotación técnica y el último, además, propiamente foucaultiana.

Por cuanto acabamos de decir, consideramos que, en lugar de la hipótesis agambeniana, debemos preferir la de Shinya Shigemi, aunque proceda de manera diferente a la nuestra y no refiera ninguna aparición del término "positividad" anterior a Les Mots et les choses. Según Shigemi, es necesario vincular los usos foucaultianos del término positividad con la obra de Compte (Shigemi, 2010, p. 47). Al respecto, es necesario señalar que no encontramos ninguna referencia textual en los escritos de Foucault, y el propio Agamben tampoco ofrece ninguna, en la que el término en cuestión aparezca vinculado a los trabajos de Hyppolite o de Hegel, o que tenga explícitamente el sentido que posee en este último. En cambio, si pensamos en la obra de Compte y en el vocabulario de su corriente de pensamiento, la proximidad con los usos foucaultianos y sus contextos resulta más que evidente, sobre todo respecto del primer significado que hemos mencionado. En el vocabulario precisamente positivista, en efecto, el término "positividad" es utilizado para hablar de aquello concreto que se opone a la vana o pura especulación. Y también respecto del tercer significado, la positividad foucaultiana comparte con la positividad positivista, permítasenos el pleonasmo, la tendencia a substituir lo absoluto por lo relativo para poder absorber dentro de una determinada sistematicidad los elementos opuestos (Shigemi, 2010, 48-49), como sucede precisamente con la noción de a priori histórico.

La afirmación acerca del origen hegeliano del término "positividad" en Foucault, a nuestro modo de ver, entonces, tiene que ser dejada de lado. En principio, el abandono de esta hipótesis no debería comprometer necesariamente la elaboración teórica propuesta en esta conferencia de Agamben y, sobre todo, la afirmación de que existe una equivalencia entre lo 
que Hegel llamaba positividad y la noción foucaultiana de dispositivo. Sin embargo, es necesario subrayar que Agamben explícitamente establece una cierta dependencia entre ellas cuando afirma: “entonces Foucault, tomando en préstamo este término [positividad] (que luego se convertirá en 'dispositivo'), toma posición respecto de un problema decisivo, que también es su problema más propio, la relación entre los individuos como seres vivientes y el elemento histórico" (Agamben, 2006, p. 11).

Según Agamben, en efecto, debemos entender por positividad en Hegel -de acuerdo a la lectura de Hyppolite que, es necesario subrayarlo, habría sido aquí el mediador entre Hegel y Foucault- el elemento histórico (reglas, ritos, instituciones, etc.) que se opone al elemento natural o simplemente racional (Agamben, 2006, pp. 10-11). Y, precisamente, cuando Hegel habla en sus escritos juveniles de la positividad de la religión cristiana, lo hace para distinguir y también oponer las formas históricas de la religión al contenido de ésta que puede deducirse de la sola razón y que coincidiría, en este caso, con los preceptos morales de la filosofía. Desde esta perspectiva, entonces, la positividad, que corresponde al elemento histórico se opone a lo racional, y también, según una observación del propio Agamben, como el elemento coercitivo a la libertad (2006, p. 10).

Las posibles relaciones entre los conceptos foucaultianos y los desarrollos del joven Hegel, como los sugeridos en esta conferencia por Giorgio Agamben, ameritan un estudio detallado del que todavía no disponemos y que trasciende ampliamente los límites de nuestro propósito actual. A modo de mera indicación, resultaría sin duda interesante vincular el juicio de Hegel acerca de la filosofía de la Aufklärung, en su Glauben und Wissen, con las consideraciones foucaultianas del capítulo noveno de Les Mots et les choses, titulado "El hombre y sus dobles". En efecto, en ambos trabajos, está el juego la nociones de finitud y subjetividad provenientes de la filosofía kantiana. También lo sería un estudio comparativo sobre algunos intereses comunes de ambos pensadores, como por ejemplo la penalidad. En este sentido, es necesario reconocer las posibilidades interpretativas que se abren a partir de la afirmación agambeniana de la relación entre la positividad de Hegel y los dispositivos de Foucault.

A pesar de ello, como ya vimos, no resulta posible sostener que Foucault haya "tomado en préstamo" el término "positividad" de Hegel y, por lo tanto, tampoco que, haciendo esto, haya querido "tomar posición respecto de un problema decisivo", la oposición 
entre historia y razón o entre coerción y libertad. Simplemente, porque estas oposiciones no se corresponden con el pensamiento de Foucault. Ni la racionalidad ni la libertad son, estrictamente hablando, elementos opuestos a la dimensión histórica de los dispositivos. Y el propio Agamben es, de algún modo, consciente de esta distancia cuando afirma que Foucault no ha querido ni "reconciliar" ni "enfatizar" el conflicto entre estos dos elementos (Agamben, 2006, p. 12).

A diferencia de Hegel, para Foucault, lo fundamental es que la razón no existe sino como historia de la razón. En este sentido, sostendrá, refiriéndose a los efectos que ha tenido en su pensamiento la lectura de Nietzsche, que hay una historia del sujeto del mismo modo que hay una historia de la razón (1994, vol. 4, p. 436). No hay un sujeto más allá de las prácticas históricas de subjetivación ni una razón independiente de las formas de racionalidad históricamente configuradas. Estrictamente, entonces, no deberíamos hablar de la razón, sino de las racionalidades. Y lo mismo debe decirse acerca de la libertad, que es pensada como una producción de los dispositivos, en especial, de los de seguridad (Foucault, 2004, p. 50).

Tocamos aquí un punto, sobre el que volveremos más adelante, y que, a nuestro modo de ver, constituye uno de los núcleos teóricos más relevantes y problemáticos de la noción de dispositivo, esto es, si existe respecto de ellos un afuera que, eventualmente, es atrapado en ellos o por ellos y, también eventualmente, puede ser liberado o liberarse de esta captura. En última instancia, si en toda filosofía de los dispositivos nos encontramos, entonces, finalmente con una ontología binaria. Esta parece ser la perspectiva agambeniana, al menos en la medida en que propone dividir todo lo que existe en dispositivos y substancias o, como se desprende también del desarrollo de su conferencia, entre dispositivos y vida o entre el lenguaje, el más antiguo de los dispositivos (Agamben, 2006, p. 22), y la vida. Entre estas dos categorías, debemos introducir sin embargo un tercer elemento, el que se produce, precisamente, en la captura de la vida por parte de los dispositivos: el sujeto, "lo que resulta del cuerpo a cuerpo entre los vivientes y los dispositivos" (2006, p. 22).

\section{Oikonomía, dispositio}

Al extender la genealogía del concepto de dispositivo al campo de la teología Agamben da un paso más que es necesario analizar. Haciendo uso de su teoría de las 
signaturas, ${ }^{5}$ aunque no se la mencione de manera expresa en esta conferencia, sostiene como ya mencionamos, que en los dispositivos foucaultianos nos encontramos con la herencia teológica de la oikonomía. Sin poder entrar en mayores detalles al respecto, ello implicaría, retomando las expresiones del autor, que estos dispositivos "pueden ser remitidos a la fractura que divide y al mismo tiempo articula, en Dios, el ser y la praxis, la naturaleza o la esencia y la operación a través de la cual él administra y gobierna el mundo de las criaturas". En este sentido, subraya, nos encontramos con una "relevancia todavía más decisiva" del concepto de dispositivo que la que puede establecerse a partir de la relación entre los dispositivos de Foucault, la positividad de Hegel o la Gestell del último Heidegger. Esta radica, según Agamben, en que, como sucede en la oikonomía teológica, es decir, en el gobierno divino del mundo, la praxis "no tiene ningún fundamento en el ser" y, por lo tanto, "los dispositivos tienen que producir su propio sujeto" (Agamben, 2006, pp. 18-19).

Dejando de lado la discusión acerca del mecanismo de las signaturas, a través del cual se establece la relación entre dispositivo y oikonomía, vemos cómo entre los elementos que componen la ontología agambeniana, las substancias y los dispositivos o el ser y la praxis, existe un factura: la praxis no se funda en el ser. Como sostendrá Agamben en Il Regno e la Gloria, donde lleva a cabo un estudio detallado de la oikonomía teológica, la divinidad es literalmente an-archós, sin principio, anárquica (Agamben, 2007, pp. 74-75). Los dispositivos nos remiten, por ello, al campo de la pura operatividad, de la "pura actividad de gobierno" (Agamben, 2006, p. 19) para administrar y controlar "los comportamientos, los gestos y el pensamiento de los hombres" (Agamben, 2006, p. 20). No hay, por ello, una substancia, sino sólo una economía del poder (Agamben, 2007, 159).

En Il Regno e la Gloria, sirviéndose de la misma teoría de las signaturas, Agamben establece una estrecha filiación entre la doctrina teológica de la providencia, a través de la cual los pensadores cristianos trataron de comprender el gobierno divino del mundo, la pura operatividad divina, y las formas modernas del gobierno económico de los hombres, en particular con el liberalismo y las democracias fundadas en el consenso. Tal como lo expresa el subtítulo, esta obra es, en efecto, "una genealogía teológica de la economía y del gobierno". Desde este punto de vista, sostiene el autor en las páginas finales del apéndice titulado "La economía de los modernos", con el que cierra este trabajo: “cuando la Modernidad abolirá el

\footnotetext{
${ }^{5}$ Acerca de esta noción, cf. el capítulo segundo, "Teoría de las signaturas", de la obra metodológica de Agamben
} del año 2008, titulada precisamente Signatura rerum. 
polo divino, la economía que deriva no se emancipa, por esto, de su paradigma providencial" (Agamben, 2007, p. 312).

También Foucault, como sabemos, desde el momento en que comienza a orientar sus investigaciones en torno a las nociones de gobierno y de gubernamentalidad, es decir, en el marco del estudio de la formación de la economía política moderna y del liberalismo, dirige su mirada hacia la historia y la literatura cristianas, en particular del período patrístico. Pero las perspectivas abiertas por estas investigaciones, a partir sobre todo del curso en el Collège de France de los años 1977-1978, Sécurité, territoire, population, es marcadamente diferente a la propuesta por Agamben. Una primera indicación al respecto la encontramos en el género de los trabajos en los que se interesan cada uno de ellos, aun cuando se trate de un mismo autor como, para citar sólo un ejemplo, San Ambrosio. En el caso de Agamben, sus referencias remiten mayormente a los tratados de carácter dogmático, en particular acerca de la Trinidad divina y del modo en que Dios gobierna providencialmente el mundo de los hombres y de las cosas; en el caso de Foucault, en cambio, a los escritos pastorales que se ocupan de la manera en que, precisamente, los pastores conducen a los creyentes. Foucault, en efecto, no está interesado en las doctrinas acerca de la naturaleza y la acción divinas, sino en el poder pastoral, en la constitución de una forma individualizante de ejercicio del poder, que constituye una de las características propias del cristianismo (Foucault, 2004a, p. 187).

Por ello, cuando Foucault se ocupa, para expresarlo de algún modo, de la herencia cristiana de la Modernidad, del poder pastoral como "preludio" (Foucault, 2004a, p. 187) de la gubernamentalidad moderna, lo hace para referirse a esas prácticas del poder pastoral que han sido reelaboradas en las formas modernas del gobierno e integradas en las estructuras de la estatalidad. Pero, para nuestro autor, ello no significa que haya habido una transferencia de la Iglesia al Estado, en la que siguen funcionando los mismos mecanismos de la primera en el segundo (Foucault, 1994, vol. 4, pp. 230-232). Sus genealogías no buscan establecer una génesis o una filiación, sino formas de multiplicación y transformación (Foucault, 2004a, p. 123). En los análisis foucaultianos, en efecto, no nos encontramos con esa hermenéutica de la securalización que, finalmente, atraviesa las nociones agambenianas de signatura y profanación. En este contexto, no resulta posible sostener, como lo hace Agamben, que el paradigma de funcionamiento del poder de las democracias modernas haya que buscarlo "en el árido latín de los tratados medievales y barrocos sobre el gobierno divino del mundo" 
(Agamben, 2007, p. 11). En términos más llanos, en lugar de remontarse a los tratados teológicos medievales, según Foucault, para describir las formas modernas del funcionamiento del poder, es necesario analizarlas en lo que ellas tienen de propio y específico.

De este modo, en relación con la afirmación de Agamben según la cual en el uso foucaultiano del término "dispositivo" continua vigente el paradigma gubernamental providencialista, nos encontramos con una dificultad semejante a la que conducían las conclusiones que el autor extraía del pretendido origen hegeliano del término "positividad". En este caso, como vimos, los dispositivos foucaultianos no suponían una distinción entre razón e historia. Ahora, respecto de la descripción de la gubernamentalidad moderna, los trabajos de Foucault no transitan los caminos de la providencia, sino el de las diferentes formas que ha tomado la constitución de una modalidad individualizante de ejercicio del poder, el poder pastoral, que, según sus análisis, precisamente en la medida en que es individualizante, se diferencia del paradigma providencial (Foucault, 2004a, p. 240).

Se trata, en definitiva, de dos genealogías diferentes de la gubernamentalidad moderna con la particularidad de que Agamben, a pesar de esta disparidad, en su conferencia del 2005 ha querido incluir la genealogía foucaultiana dentro de su propia genealogía o, en otros términos, no sólo ha llevado a cabo una genealogía teológica de la economía y del gobierno, sino también una genealogía teológica de la genealogía foucaultiana. Y, a decir verdad, más allá de todas las dificultades que hemos señalado, con algunos buenos motivos o, desde la perspectiva del propio Agamben, siguiendo uno de los principios metodológicos que ha guiado frecuentemente sus investigaciones, esto es, la capacidad que tiene cualquier obra de ser desarrollada, según la expresión de Feuerbach retomado por el autor, su Entwicklungsfähigkeit (Agamben, 2008, p. 8).

En efecto, ya en Homo sacer. Il potere sovrano e la nuda vita, Agamben señala que, en los desarrollos foucaultianos acerca de la biopolítica, permanecía irresuelto u oculto el punto de entrecruzamiento entre los mecanismos jurídico-institucionales y los desarrollos del biopoder, es decir, aquellos a través de los cuales el derecho entra en relación con la vida (Agamben, 1995, p. 8-9). Para resolver esta cuestión, como sabemos, Agamben se sirve de la noción de excepción soberana entendida como relación de exclusión-inclusiva (Agamben, 1995, p. 10). En un contexto diferente, ya no centrado en el concepto de soberanía sino de 
economía, esta tarea es continuada en Il Regno e la Gloria, aunque el autor no sea igualmente explícito al respecto. Pero ahora, para esclarecer ese punto oscuro, ya no se recurre a la noción de excepción, sino de la Gloria, entendida como la dimensión celebrativa y aclamativa del poder que articula, según Agamben, los dos polos de la máquina política de Occidente, el reino o la soberanía por un lado y el gobierno por otro.

\section{Profanación y veridicción}

Como decíamos al inicio de nuestro trabajo, resulta casi evidente vincular esta conferencia de Giorgio Agamben de 2005 con aquella homónima de Gilles Deleuze en 1988. En cierto sentido, es la repetición de ese gesto intelectual. En el caso de Deleuze, ella cierra un ciclo de escritos acerca de la noción de dispositivo iniciado en 1975 con la reseña de Surveiller et punir titulada, "Écrivain non: un nouveau cartographe", ${ }^{6}$ donde Deleuze se muestra particularmente entusiasta respecto de las posibilidades teóricas de esta noción y subraya las correspondencias entre sus categorías y las de Foucault. La posición de Deleuze cambiará muy poco después con la aparición de La Volonté de savoir. En un breve pero denso texto, escrito en 1977 pero publicado recién 1994, "Désir et plaisir", ${ }^{7}$ Deleuze dirige una serie de críticas a esa reciente publicación de Foucault. Dos de ellas merecen ser subrayadas en relación con los objetivos del presente trabajo. En primer lugar, la que concierne a lo que Deleuze denomina líneas de fuga, es decir, la posibilidad de modificar los dispositivos, de escapar de ellos. En segundo, los cuestionamientos a Foucault a propósito de la reintroducción del concepto de verdad (Deleuze, 2003, pp. 113, 116). Estas mismas crítica serán retomadas, algunos años más tarde, en una larga nota de Mille plateaux (Deleuze, 1980, pp. 175-176, nota 36). En el marco de este ciclo de escritos, la conferencia de 1988 puede ser vista como un retorno a las posiciones sostenidas en la reseña de 1975. Y también como una reconciliación de Deleuze con el pensamiento de Foucault, sobre todo en la medida en que encuentra ahora en su pensamiento la posibilidad de lo actual, es decir, de introducir la novedad en el juego de los dispositivos (Deleuze, 2003, pp. 322-323).

\footnotetext{
${ }^{6}$ Reeditado en el libro que Deleuze consagrará a Foucault bajo el título "Un nouveau cartographe” con algunas modificaciones (Deleuze, 1986, pp. 31-51).

${ }^{7}$ Actualmente reeditado en Deleuze, 2003, pp. 112-122.
} 
Entre otras razones, por una cuestión de espacio, no cabe dentro de nuestro propósito ahondar más en detalle las relaciones entre las conferencias de Deleuze y Agamben. Nos hemos limitado a subrayar apenas estos dos aspectos, la posibilidad de escapar de las capturas con que los dispositivos atrapan la vida (para servirnos del vocabulario de Agamben) y la cuestión de la verdad, porque éstos remiten a las dos nociones de las que nos ocuparemos a continuación, profanación y veridicción, y nos permiten situarlas en un contexto intelectual más amplio.

Volviendo a nuestro autor, Agamben, luego de haber hecho del concepto de dispositivo una de las dos categorías ontológicas en las que puede dividirse todo lo que existe, propone también lo que bien podría denominarse una política de los dispositivos, en la que el lugar de las líneas de fuga, en las consideraciones de Deleuze, es ocupado ahora por la noción de profanación, presentada precisamente como un contradispositivo (y a la que el autor dedica un entero trabajo el mismo año de su conferencia que aquí está en cuestión, titulado precisamente Profanazioni). Agamben, en efecto, concibe en términos de sacrificio o separación ritual a la operación mediante la cual los dispositivos capturan a la vida. Como hace notar el autor, ya en el ámbito del derecho romano se denomina profanación a la operación inversa: "la profanación es el contradispositivo que restituye al uso común lo que el sacrificio había separado y dividido" (Agamben, 2006, p. 28). Como en Deleuze, además, esta operación remite a un "punto de fuga", en este caso, de toda política (Agamben, 2006, p. 35).

En su conferencia, Agamben no nos ofrece muchas más precisiones, excepto respecto de la relación entre subjetividad y verdad o, mejor, no-verdad. En la fase actual del capitalismo -sostiene- los dispositivos, más que a la subjetivación, dan lugar a procesos de desubjetivación, donde no está en juego la verdad del sujeto, sino su no-verdad, hasta el punto de producir, en definitiva, formas espectrales: "un número a través del cual puede ser, eventualmente, controlado", "una máscara", "la inclusión en el cálculo de un índice" (Agamben, 2006, p. 31).

Con la intención explícita de extender al máximo la noción de dispositivo mucho más allá de Foucault, Agamben enumera una serie de operaciones que pueden ser consideradas como las diferentes formas del sacrificio, de la separación ritual: "llamaré dispositivo literalmente [sostiene] a cualquier cosa que tenga de algún modo la capacidad de capturar, orientar, determinar, interceptar, modelar, controlar y asegurar los gestos, las conductas, las 
opiniones y los discursos de los seres vivientes" (Agamben, 2006, pp. 21-22). El producto de estas operaciones, como ya hemos señalado, es la subjetividad, actualmente espectral.

Ahora bien, respecto del más antiguo de estos dispositivos, ${ }^{8}$ el lenguaje, Agamben precisa, en Profanazioni, que "una subjetividad se produce donde el viviente, encontrando el lenguaje y poniéndose en juego en él sin reservas, exhibe en un gesto la propia irreductibilidad al lenguaje" (Agamben, 2005, 81). Y poco después, el mismo año en que publica su obra metodológica sobre las signaturas, la operación sacrificial, la sacratio, del más antiguo de los dispositivos es objeto de un entero escrito, titulado precisamente $\mathrm{Il}$ sacramento del linguaggio. Una archeologia del giuramento (2008). El juramento, en efecto, es visto como la forma arqueológicamente originaria de la sacratio que, para el viviente, tiene lugar en el lenguaje (Agamben, 2008b, p. 90). "Bendiciendo y maldiciendo, jurando y perjurando", sostiene Agamben, el hombre "se pone en juego en la palabra" (Agamben, 2008b, p. 98). Hacia el final de esta obra, la filosofía es presentada como la operación inversa de la sacratio del juramento, como el cuestionamiento de la primacía de los nombres (Agamben, 2008b, p. 98) o, si queremos, de un "modelo performativo de la verdad" (Agamben, 2008b., 76). En definitiva, aunque el autor no se sirva explícitamente de estos términos, la filosofía se convierte, entonces, en una forma de profanación del lenguaje, en un contradispositivo.

Entre las nociones de lo que se ha denominado el último Foucault, una ocupa, sin lugar a dudas, un lugar de privilegio: parresía, el decir franco, el decir veraz. Dos cursos enteros, precisamente los últimos en el Collège de France, le están dedicados: Le Gouvernement de soi et des autres y Le Courage de la vérité. Tal es su importancia que Foucault define a la filosofía como "el movimiento de la parresía" (Foucault, 2008, p. 322). Al igual que en las reflexiones agambenianas que acabamos de exponer, la problemática de la parresía no es otra que la de la relación entre vida y lenguaje, es decir, la subjetividad, el modo en el que, quien habla, expone su vida en el lenguaje. Y también aquí, como sucede en Agamben, se recurre a un modelo de verdad inverso al de la perfomatividad, que Foucault denomina "dramática del discurso", en la medida en que no son las condiciones institucionales o rituales las que rigen la verdad del enunciado sino la situación real de quien habla, que afecta y modifica el valor y el sentido del enunciado (Foucault, 2008, pp. 64-65).

${ }^{8}$ Es cierto que, en el texto de la conferencia, el adverbio forse, quizás, acompaña a la expresión "el más antiguo de los dispositivos". Sin embargo, ello es taxativamente afirmado en Agamben, 2005, p. 81. 
En la larga historia foucaultiana de la parresía que recorren los dos cursos que hemos apenas mencionado, los cínicos, despojados de toda la carga negativa con la que habitualmente se los aborda, son considerados por Foucault como una categoría o una figura transhistórica de la subjetividad occidental (Foucault, 2009, 161), en la medida en que, en ellos, la propia vida, y no sus doctrinas, se presenta como una manifestación de la verdad. Ellos representan, según la expresión del propio Foucault, "la producción de la verdad en la forma de la vida misma" (2009, p. 200).

Desde esta perspectiva, aplicando ahora a Agamben el principio metodológico del que frecuentemente se sirve y que resume en el concepto de Feurbach Entwicklungsfähigkeit, podría sostenerse, sin dudas, que la noción agambeniana de profanación y la foucaultiana de veridicción parresíastica forman parte de esa misma política de los contradispositivos que busca en la experiencia no performativa del lenguaje una correlación sin restos entre vida y subjetividad.

\section{Referências}

AGAMBEN, G. (1995). Homo sacer. Il potere sovrano e la nuda vita. Torino: Einaudi. . (2005). Profanazioni. Roma: Nottetempo. . (2006). Che cos'è un dispositivo. Roma: Nottetempo. . (2007). Il Regno e la Gloria. Per una genealogia teologica dell'economia e del governo. Vicenza: Neri Pozza. . (2008a). Signatura rerum. Sul metodo. Torino: Bollati Boringhieri.

$\overline{\text { Laterza. }}$ (2008b). Il sacramento del linguaggio. Una archeologia del giuramento. Bari:

Castro, E. (2011). Diccionario Foucault. Temas, conceptos, autores. Buenos Aires: Siglo XXI Editores.

Deleuze, G. y Guattari, F. (1980). Mille plateaux. Capitalisme et schizophrénie 2. Paris: De Minuit.

Deleuze, G. (1986). Foucault. Paris: De Minuit. . (2003). Deux régimes des fous. Paris: De Minuit

Foucault, M. (1961). Folie et déraison. Histoire de la folie à l'âge classique. Paris: Plon. Gallimard. (1966). Les mots et les choses. Une archéologie des sciences humaines. Paris: 
. (1969). L’Archéologie du savoir. Paris: Gallimard.

. (1976). La Volonté de savoir. Histoire de la sexualité 1. Paris: Gallimard.

. (1994). Dits et écrits (4 vols.). Paris: Gallimard.

. (2003). Le Pouvoir psychiatrique. Cours au Collège de France, 1973-1974. Paris:

Gallimard-Seuil

. (2004a). Sécurité, territoire, population. Cours au Collège de France, 1977-1978.

Paris: Gallimard-Seuil

. (2004b). Naissance de la biopolitique. Cours au Collège de France, 1978-1979.

Paris: Gallimard-Seuil.

. (2008). Le Gouvernement de soi et des autres. Cours au Collège de France, 19821983. Paris: Gallimard-Seuil

. (2009). Le Courage de la vérité. Cours au Collège de France, 1983-1984. Paris: Gallimard-Seuil

Hegel, G. W. F. (1962). Glauben und Wissen: oder die Reflexionsphilosophie der Subjektivität in der Vollständigkeit ihrer Formen als Kantische, Jacobische und Fichtesche Philosophie. Hamburgo: Meiner.

Shigemi, Sh. (2010). Sur la "positivité" chez Foucault. Conferencia obtenida el 1 de enero de 2015 en: http://www.gcoe.lit.nagoya-u.ac.jp/eng/result/pdf/05 Shigemi.pdf. 